\title{
Current State of Women in Academic Surgical Subspecialties: How a New Metric in Measuring Academic Productivity May Change the Equation
}

\author{
Alison M. Fecher, M.D., Nakul Valsangkar, M.D., Teresa M. Bell, Ph.D., Megan E. Lisy, M.D., \\ Grace S. Rozycki, M.D., M.B.A., and Leonidas G. Koniaris, M.D., M.B.A. \\ Department of Surgery, Indiana University, Indianapolis, Indiana
}

In the August 2016 issue of the Journal of the Am Coll Surg, our group reported the differences between male and female academic surgeons by faculty rank, academic output, and NIH funding rates using data of faculty from the 55 departments of surgery with the most National Institutes of Health funding. ${ }^{1}$ Overall, metrics from 4015 surgical faculty members were examined. The objectives of the study were to identify potential barriers that might be preventing the academic success of all surgical faculty members. Men comprised 76.9 per cent (3087) and females comprised 23.1 per cent (928) of the faculty we could identify. Male surgical faculty also comprised a greater fraction of older surgeons.

Overall, male cumulative scholarly output was higher than that of females as measured by total publications ( $43 \pm 97.5$ vs $1958, \mathrm{p}<0.05)$ and total citations $(723 \pm 3322$ vs 317 $\pm 1,774, \mathrm{p}<0.05$ ). In terms of gender-based differences in academic rank, female surgical faculty members were far more likely to be represented at lower academic levels. Overall a higher proportion of women faculty are at junior faculty ranks with 49.7 per cent of the female surgical faculty at the assistant professor rank, compared with only 31.8 per cent of male faculty. An equal proportion of male and female surgical faculty were at the associate professor level (27\%); with only 22.7 per cent of female surgical faculty being at full professor rank compared with 41.2 per cent of males. This is disproportionate even accounting for the differences in the demographics of medical students and residents five and 10 years prior. ${ }^{2,3}$ At higher academic ranks, the publications and citations for women were similar to those of their equivalently ranked male counterparts. Gender-based differences in leadership positions and academic degrees were also found. Female surgical faculty members were, in fact, half as likely to be in divisional leadership positions.

The fraction of women by surgical subspecialty examined also differed. Cardiothoracic surgery had the lowest fraction of female full professors (11\%). Among thoracic surgeons, 22 per cent of the female faculty were full professors, compared with 48 per cent male surgical faculty. These numbers were lower for female cardiac surgery professors. The findings of lower percentages of women at the rank of full professor were observed for other surgical specialties as well, including both vascular surgery and surgical oncology. ${ }^{1}$

Address correspondence and reprint requests to Leonidas G. Koniaris, M.D., M.B.A., F.A.C.S., 545 Barnhill Drive EH 511 Indianapolis, IN 46202.1koniari@iu.edu. 
The data from this analysis are supported by other analyses performed by a variety of other approaches and across different medical specialties or other professions. Nonnemaker, for example, analyzed the promotion trends across all disciplines and departments of academic medicine and evaluated differences in medical student gender and its effect on promotion and academic position by decade. ${ }^{4}$ The article, published in the $\mathrm{N}$ Engl $\mathrm{J}$ Med, showed that there were higher numbers of female medical students graduating than men in the 1980s. However, overall promotion of females from assistant professor to associate professor, as observed in our data set, was much less common for women. Similarly, female faculty advancing from associate to full professor deviated from the predicted values considerably. In the same group, the percentage of male full professor faculty was 34 per cent, compared with only 12 per cent for female faculty, regardless of department. This suggests that even accounting for differences in demographics between the graduating medical students and junior residents, the promotion of women in academic surgery has been slow.

To evaluate whether female assistant and associate professors were being promoted with similar academic measures, the scholarly output for each academic rank was broken down into deciles. Comparisons of these deciles between genders for each corresponding rank did not indicate an increased concentration of women at lower ranks with higher publication/ citation numbers. For example, 22 publications for an individual general surgery faculty member who is an assistant professor would place that person below the 70th percentile among men and above the 70th percentile among women.

It is critical to understand the underlying reasons for these significant disparities in rank and in leadership positions and to determine ways to address them. In our study, we found that women as a group tended to publish fewer articles than men. Publications authored by female surgical faculty, however, were more likely to be cited, particularly soon after publication. This suggests these publications had greater impact and overall were of higher quality. This improved quality can be measured by calculating the median number of threeyear citations per total publications. We have termed this measure "academic velocity" or "V." The advantage of this metric is that it captures the contributions of upcoming researchers who have published actively in the last three to five years, whereas somewhat discounting publications accrued many years ago. Ostensibly, the negative effects of the practices of guest authorships that were more prominent almost a decade ago are also attenuated with the use of this metric. It may not be much of a surprise then that with regard to this metric woman score slightly higher than men (5.5 vs 4.5$)$. Its use may even the playing field in that it helps to capture the current state of a surgeon's academic productivity, potential, and trajectory; parameters in which gender differences do not exist. Current evaluation practices do not always take into account the impact and quality of publications authored. The use of the "V" metric may help division chiefs and chairs in making decisions concerning the hiring and promotion of women and, ultimately, may be a better evaluation of academic productivity than unweighted publication counts.

Work by Nattinger and coworkers have identified decreased resource allocation as a significant contributor to the observed academic disparity women face. Female surgeons have been found to have fewer academic resources; poorer clinical support infrastructure; less secretarial support; smaller laboratory space and office space; and less grant assistance, 
protected research time, and other research assistance. ${ }^{5}$ More importantly, this observed lack of work infrastructure is likely associated with reduced mentorship opportunities.

The mentor/mentee relationship is an integral component to both personal and professional development, as it plays a major role in building confidence and establishing the leadership skills necessary to gain recognition in academic society and health systems. Historically, the lack of women in leadership roles has potentially hampered the academic advancement of women overall. This lack of female representation in leadership positions may have repercussions by reducing the policies and approaches that prevent obstacles for junior ranked women in career advancement. This problem is also not unique to medicine. A review of Fortune 500 female executive officers reveals that only 15 per cent of women fill this role and only 17 per cent advance to become board members.

The barriers to success for women may also be considered in terms of the perception of their availability. For instance, a gender study group from a major academic institution found that 10 per cent of female and 45 per cent of male academic physicians have spouses who are not employed outside of the home. ${ }^{6,7}$ This study suggested that female physicians spend considerably more hours on parenting and have less help with home and family duties than their married male counterparts. This leaves a disproportionate amount of family and household duties to the full-time female physician. With these added responsibilities at home, a female surgeon may be perceived as being too busy or too distracted to take on a leadership role. Nevertheless, a female surgeon may be applauded for her multitasking ability, but her career role may become one of service rather than leadership.

Ultimately there are many different avenues for further research and work by departments of surgery to make sure that all faculty members can succeed. Many have reported we are on the cusp of increasing the number of women in leadership roles whose qualifications ensure that they should be in these positions and hopefully as they ascend to these positions, they will also support other women deserving of promotion. The use of academic metrics that favor recent and higher impact publications can help identify academically strong individuals. Thoughtful allocation of resources to provide women with equal protected time and funding for research at an earlier career stage, and enhanced participation of women in mentorship programs at all academic levels are needed. Furthermore, understanding barriers that women are more likely to face in their careers, changing the mindset among surgical faculty members to make the surgical workplace inclusive, and helping those in leadership positions to not only be accommodating but support the measures needed to allow women to excel in academic surgical specialties are needed.

\section{References}

1. Valsangkar N, Fecher AM, Rozycki GS, et al. Understanding the barriers to hiring and promoting women in surgical sub-specialties. J Am Coll Surg. 2016; 223:387-98. [PubMed: 27109779]

2. Cochran A, Hauschild T, Elder WB, et al. Perceived gender-based barriers to careers in academic surgery. Am J Surg. 2013; 206:263-8. [PubMed: 23414631]

3. Beasley BW, Wright SM, Cofrancesco J, et al. Promotion criteria for clinician-educators in the United States and Canada. A survey of promotion committee chairpersons. J Am Med Assoc. 1997; 278:723-8. 
4. Nonnemaker L. Women physicians in academic medicine: new insights from cohort studies. N Engl J Med. 2000; 342:399-405. [PubMed: 10666431]

5. Tesch BJ, Wood HM, Helwig AL, et al. Promotion of women physicians in academic medicine. Glass ceiling or sticky floor? J Am Med Assoc. 1995; 273:1022-5.

6. CrossleyAD. On the Cutting Edge? A Look at the "Medical" Gender Gap for Women Surgeons [Internet]Clayman Institute for Gender Research; 2015

7. Waldfogel J. Understanding the 'Family gap' in pay for women with children. J Econ Perspect. 1998; 12:137-56. 